\title{
An improved algorithm for PMU assisted islanding in smart grid
}

\author{
Mohd Rihan, Mukhtar Ahmad, Mohammad Anas Anees*
}

Aligarh Muslim University, Aligarh - 202002, India

\begin{abstract}
In case of a cascading failure, the power grid splits into uncontrolled islands before descending into blackout. Intelligent islanding is an important method to reduce the risk of blackout by preventing propagation of disturbance to other part of the system. Phasor measurement units are now integral part of a power system are very effective in detection of islanding. This paper presents an algorithm for power system intentional islanding to avoid the propagation of faults to healthy sections of the network in case of disturbances by using the synchrophasors measurement. The proposed algorithm has wider applicability and performs better in terms of network observability.
\end{abstract}

Keywords: Smart grid, islanding, synchrophasors, PMU, microgrid

\section{Introduction}

The ever increasing power demand, high quality power requirements of a predominantly digital society, and rising environmental concerns are making it difficult to operate the grid in a secure and satisfactory way. In a Highly stressed grid, a contingency involving the loss of several important transmission lines may trigger cascading events and eventually lead to a black out. Such contingencies are not common but may arise due to storms or tripping of large generator or faults in large capacity transmission line. Among different protection schemes, controlled separation (or islanding) is considered the last line of defence to stabilize the system by actively separating the whole power system into two or more pre-designed islands. For this purpose intentional islanding can be used with proper designed islands. By tripping the weak tie lines connecting different islands when a severe disturbance is detected, the local disturbance can be effectively confined to only one island by proper control.

Apart from better real-time monitoring, PMU deployment will also help in maintaining proper operation of islands created to prevent a blackout. Another application of PMUs in islanding is the utility of these units in maintaining the observability of individual islands [1].

An algorithm for intentional islanding of a power grid satisfying various islanding constraints was presented in [2], [3]. However the algorithm is not applicable to all networks and failed to keep the islands observable in some cases, as shown in the present paper. This paper presents an improvement in the islanding algorithms such that it is applicable to all the networks. Moreover the algorithm under consideration was based on the unrealistic assumption of an infinite channel capacity of available PMUs. But in practice the PMU has a limited channel capacity; in fact it has been shown in the earlier studies that usually it is neither economical nor necessary to use PMUs with a channel limit of more than 3 or 4 [4]. Keeping this fact in view, effect of PMU channel capacity on islanding scheme has also been investigated in the present work.

To compare the islanding options an index 'weighted observability percentage index (wop)' was also defined in the original algorithm. A higher value of wop represents more number of important buses is observable. The improved algorithm reported here provides a higher value of wop as compared to earlier

\footnotetext{
* Manuscript received October 22, 2015; revised January 18, 2016.

Corresponding author. Tel.: +919219605655; E-mail address: m.rihan.ee@amu.ac.in.

doi: $10.12720 /$ sgce.5.1.29-40
} 
reported algorithm.

\section{PMU Assisted Intentional Islanding}

A PMU placed at a given bus is capable of measuring the voltage phasor of the bus as well as the phasor currents for some or all the lines incident to that bus. Thus, the entire system can be made observable by placing PMUs at strategic buses in the system. The objective of the PMU placement problem is to accomplish this task by using a minimum number of PMUs. For an $\mathrm{n}$ bus system, the PMU placement problem can be formulated using integer programming method as follows [5]:

$$
\min \sum_{i}^{n} w_{i}, x_{i}
$$

where

$$
\text { s.t. } f(x) \geq \mathbf{b}
$$

$\mathbf{X}$ is a binary decision variable vector, whose entries are defined as:

$$
x_{i}=\left\{\begin{array}{l}
1 ; \text { if a PMU is installed at bus } i \\
0 ; \text { otherwise }
\end{array}\right.
$$

$w_{1}$ is the cost of PMU installed at bus i. If costs of all PMUs are equal, all the entries in $\boldsymbol{w}$ vector will be $1 . \mathbf{f}(\mathbf{X})$ is a vector function, whose entries are non-zero if the corresponding bus voltage is solvable using the given measurement set and zero otherwise.

In general, $\mathrm{f}(\mathbf{X})$ can be found as:

$$
f(X)=A . X
$$

$\mathbf{A}$ is the system connectivity matrix. Each row of $\mathbf{A}$ corresponds to a bus of the system and each column corresponds to the buses connected to that bus i.e.

$$
A_{\mathrm{i} j}=\left\{\begin{array}{l}
1 ; \text { if } j \text { th bus is connected to ith bus } \text { OR } i=j \\
0 ; \text { otherwise }
\end{array}\right.
$$

b vector represents how many times a bus is needed to be observable. It tells the required redundancy level of measurements for a bus. If any element of $\mathbf{b}$ matrix is greater than one, it simply means that the corresponding bus is observable from more than 1 direction. In present work, all the entries of $b$ are taken as 1 .

Hence, equation (1) is solved to obtain the solution vector $\mathbf{X}$ which gives the required PMU locations.

The PMU assisted islanding algorithm assumes that the minimum number of PMUs required to ensure system observability are installed in the system. An important consideration in intentional islanding is identifying the instant at which the intentional islanding must be implemented. Synchronized measurements available from PMUs deployed in the power grid can be used for assessing the system state and taking the decision for intentional islanding. If the islands are formed judiciously such that a sufficient number of PMUs are installed in the individual islands, these units will help in maintaining the observability of individual islands. The algorithm presented ensures that observability of individual islands is not lost after execution of intentional islanding.

In PMU assisted Islanding, following a fault the whole system is split into observable Islands such that each Island is being monitored by PMUs and have sufficient observability. By doing so, the fault propagation among various Islands (parts of the sectionalized system) is prevented. Such a sectionalized system can then be restored gradually to normal operation once the fault is cleared thereby reducing the probability of a Grid blackout. The algorithm proposed in [2] and [3] is modified in this work for better observability of Islands and channel limit considerations are also incorporated. The original algorithm is explained briefly here and the modifications proposed are explained in the following section. 
The Maximum number of Islands is limited by the minimum of the loads, PMUs and black start generators. For an $n$ bus system this can be formulated as:

$$
\mathrm{m}=\min \left(\sum_{\mathrm{i}=1}^{\mathrm{n}} \mathrm{b}_{\mathrm{i}} \sum_{\mathrm{i}=1}^{\mathrm{n}} 1_{\mathrm{i}} \sum_{\mathrm{i}=1}^{\mathrm{n}} \mathrm{f}_{\mathrm{i}}\right)
$$

where

$$
\begin{aligned}
\mathrm{b}_{\mathrm{i}} & =\left\{\begin{array}{l}
1 ; \text { if ith bus has a blackstart generator } \\
0 ; \text { otherwise }
\end{array}\right. \\
\mathrm{l}_{\mathrm{i}} & =\left\{\begin{array}{l}
1 ; \text { if ith bus has a load } \\
0 ; \text { otherwise }
\end{array}\right. \\
\mathrm{f}_{\mathrm{i}} & =\left\{\begin{array}{l}
1 ; \text { if ith bus has a PMU } \\
0 ; \text { otherwise }
\end{array}\right.
\end{aligned}
$$

Having known the limiting factor $\left(\mathrm{b}_{\mathrm{i}}, \mathrm{l}_{\mathrm{i}}\right.$ or $\left.\mathrm{f}_{\mathrm{i}}\right)$, Initial Island matrix $\mathrm{I}^{1}$ is formed as:

$$
\mathrm{I}^{1}=\mathrm{G}=\left[\begin{array}{ccc}
\mathrm{g}_{11} & \cdots & \mathrm{g}_{1 \mathrm{n}} \\
\vdots & & \vdots \\
\mathrm{g}_{\mathrm{m} 1} & \cdots & \mathrm{g}_{\mathrm{mn}}
\end{array}\right]=\left[\begin{array}{ccccc}
\mathrm{b}_{1} & \mathrm{~b}_{2}\left(1-\mathrm{g}_{11}\right) & \mathrm{b}_{3}\left(1-\mathrm{g}_{11}\right)\left(1-\mathrm{g}_{12}\right) & \ldots & \mathrm{b}_{\mathrm{n}}\left(1-\mathrm{g}_{11}\right) \ldots\left(1-\mathrm{g}_{1(\mathrm{n}-1)}\right) \\
0 & \mathrm{~b}_{2}\left(1-\mathrm{g}_{12}\right) & \mathrm{b}_{3}\left(1-\mathrm{g}_{12}\right)\left(1-\mathrm{g}_{22}\right) & \ldots & \mathrm{b}_{\mathrm{n}}\left(1-\mathrm{g}_{1 \mathrm{n}}\right)\left(1-\mathrm{g}_{22}\right) \ldots\left(1-\mathrm{g}_{2(\mathrm{n}-1)}\right) \\
. & \cdot & . & . & \mathrm{b}_{\mathrm{n}}\left(1-\mathrm{g}_{1 \mathrm{n}}\right)\left(1-\mathrm{g}_{2 \mathrm{n}}\right)\left(1-\mathrm{g}_{33}\right) \ldots .\left(1-\mathrm{g}_{3(\mathrm{n}-1)}\right) \\
\cdot & \cdot & . & . & \mathrm{b}_{\mathrm{n}}\left(1-\mathrm{g}_{1 \mathrm{n}}\right) \ldots\left(1-\mathrm{g}_{(\mathrm{n}-1) \mathrm{n}}\right)
\end{array}\right]
$$

If number of black start generators i.e. $b_{i}($ where $i=1,2,3 \ldots n)$ are not the minimum term in (5) then it is replaced by the minimum term in (5). Island matrix is a $\mathrm{m} \times \mathrm{n}$ matrix where $\mathrm{I}_{\mathrm{ij}}=1$ if bus $\mathrm{j}$ is in $\mathrm{i}^{\text {th }}$ island. Otherwise, $\mathrm{I}_{\mathrm{ij}}=0$.

The "unmodified islands' matrix" for the next stage can be derived as:

$$
\mathbf{I}^{\prime(\mathbf{k}+\mathbf{1})}=\mathbf{I}^{\mathbf{k}} \times \mathbf{A}
$$

To obtain $\mathbf{I}^{\mathbf{k}}$ matrix from $\mathbf{I}^{\mathbf{k} \mathbf{k}}$ a column by column check is performed to see whether a same bus is assigned to different islands i.e. whether there are more than one non-zero elements in a column. Based on the following five criteria it is decided to which island this common bus is moved.

1. Central Bus Criterion: Each Island can have only one central bus. Non-zero elements corresponding to central bus of other island is removed.

2. History Criterion: If a bus is allocated in an island in previous stage, it cannot be allocated to other islands in later stages. Such non-zero entries should be removed.

3. Least Buses Criterion: If a bus is allocated to more than one islands and it is the first time they are appearing in these islands then such buses are moved to the islands of least number of buses. This is done considering original system having transformers and buses connected to them (and not the equivalent network as described earlier).

4. Multiple-Connectivity Criterion: If in the previous criterion, the two islands have equal buses, then the common bus is moved to the island where the bus has larger entry. Because it shows that the common bus has more number of buses connected to it in that island via boundary lines.

5. Shorter boundary line Criterion: If in the previous criterion, the value of entry is same in all islands, then the common bus is moved to an island that has a shorter boundary line connected to that bus to facilitate restoration.

The above process is repeated till all the buses are allocated to the island matrix without any common bus to different islands. Having formed the island matrix, the next step is to perform observability analysis and check various constraints for the islands formed.

In order to maintain integrity of individual islands, static and dynamic stability of each island should be maintained. An islanding scheme satisfying this requirement is termed as proper islanding. A proper 
islanding scheme should satisfy the following constraints [6]:

1. Integrity constraint: There should be interconnection between all the buses inside an island.

2. Synchronization constraint: All the generators in an island should be synchronized.

3. Power balance constraint: This constraint requires that the power generated by the generators in a particular island should be almost equal to the total load in the island.

4. Line limit constraint: The transmission lines should not be loaded above their thermal and steady state limits.

In order to apply the strategy of intentional islanding, three tasks should be carried out sequentially.

1. Recognizing the proper instant at which applying intentional splitting is inevitable otherwise the system will be separated into uncontrolled islands.

2. Identifying the proper islands for intentional network separation such that each island will be able to preserve its power balance and stability.

3. Implementation of the planned islanding scenario in a proper way without any dynamic and transient consequence causing large oscillation and instability for islands.

\subsection{Power system restoration constraint}

The first constraint is as follows:

$$
\sum_{j=1}^{\mathrm{J}_{\mathrm{i}}} \mathrm{P}_{\mathrm{Gj}}^{\mathrm{min}} \leq \sum_{\mathrm{j}=1}^{\mathrm{J}_{\mathrm{i}}} \mathrm{P}_{\mathrm{Lj}} \quad \mathrm{i}=1,2,3 \ldots \mathrm{m}
$$

where $J_{i}$ is the number of buses in island $i, P_{G j}^{\min }$ is the minimum output power of generator $j$ and $\mathrm{P}_{\mathrm{Lj}}$ is the demand of bus $\mathrm{j}$.

If there is any island for which (8) did not hold, non-central buses in neighbouring islands connected via boundary lines to the island are searched which have enough demand to satisfy the constraints. Such buses are brought in the island provided that the constraints of the neighbouring island do not violate due to elimination of such buses.

\subsection{Observability constraint of island}

For observability analysis of islands, vector $\mathbf{0}$ is found as:

$$
\mathbf{o}=\mathbf{A}_{\mathrm{I}} \times \mathbf{f}
$$

where $\mathbf{f}$ is given in (3) and $A_{I}$ is the connectivity matrix of the network as defined in (4) with the all entries corresponding to boundary buses made zero. $\mathbf{o}$ is a $1 \times \mathrm{n}$ vector in which zero elements indicate unobservable buses.

If zero entries are found in $\mathbf{0}$ matrix, matrix $\mathbf{U}$ is formed as:

$$
\mathbf{U}=\mathbf{A}_{\mathrm{u}} \times \mathbf{P I M}
$$

where $\mathbf{A}_{\mathrm{u}}$ is same as $\mathrm{A}_{\mathrm{I}}$ matrix containing only the rows corresponding to unobservable buses as found by vector $\mathbf{0}$. Hence $\mathbf{A}_{\mathrm{u}}$ is a $\mathrm{u} \times \mathrm{n}$ matrix where $\mathrm{u}$ is the number of unobservable buses. PIM matrix is defined as:

$\mathrm{PIM}=\left[\begin{array}{llll}\mathrm{f}_{1} & & & \\ & \mathrm{f}_{2} & & \\ & & \ddots & \\ & & & \mathrm{f}_{\mathrm{n}}\end{array}\right]_{\mathrm{n} \times \mathrm{n}}$

$$
\text { Or PIM = diagonal (f) }
$$

Each row in $\mathbf{U}$ matrix represents unobservable bus and columns of nonzero entry represent the buses in 
neighboring islands at which PMUs are installed and can make the unobservable bus observable. For example in $\mathbf{U}$ matrix shown below, bus 1 can be made observable by PMU at bus $n$, bus 2 by PMU at buses 3 and n, bus 3 by PMU at bus 3 .

$$
\mathbf{U}=\frac{1}{6} \underset{6}{6}\left[\begin{array}{lllll}
0 & 0 & 0 & \ldots & 1 \\
0 & 0 & 1 & \ldots & 1 \\
0 & 0 & 1 & \ldots & 0
\end{array}\right]
$$

Weighted observability percentage index (wop): This index is the measure of overall island observability. Its value should be greater than 0.9 for each island for sufficient observability to ensure safe system restoration. For ith island it can be defined as:

$$
\operatorname{wop}_{1}=\sum_{j=1}^{h_{1}} \frac{w_{1} \times o_{1}}{w_{1}}, i=1,2, \ldots m
$$

where $o_{j}$ is the jth entry of $o$ and $w_{j}$ is the observability weight of bus $j$ defined as:

$$
w_{j}=\left\{\begin{array}{l}
1, \text { if } \text { a bus } j \text { has a generator } \\
0.9, \text { if a bus } j \text { has aload } \\
0.8, \text { otherwise }
\end{array}\right.
$$

If more buses are observable, wop will be more. Also, if more important buses are observable, wop will be more.

\subsection{Managing the unobservable buses}

After forming the initial islands the next step is to shift the unobservable buses to make them observable. Three conditions may arise.

2.3.1 The unobservable bus is not a central bus

For this case the unobservable bus is moved to the neighbouring island where a PMU installed bus can make this bus observable. Buses connected to unobservable buses via transformers with move along with the unobservable buses as they cannot be put in separate islands. While doing so, it is ensured that buses connected via transformers to the unobservable bus must be observable in the new island. Island selectivity matrix decides in which island the unobservable bus should be moved which is given by:

$$
\mathbf{I}_{\mathbf{S}}=\left(\mathbf{I} \times \mathbf{A}_{\mathbf{u} 2} \times \mathbf{P I} \mathbf{M}^{\mathrm{T}}\right)^{\mathrm{T}} \times \mathbf{M}_{\mathbf{d i a g}}
$$

where, $\mathbf{A}_{\mathrm{u} 2}$ is the $\mathbf{A}_{\mathrm{u}}$ matrix having only the rows corresponding to non-central unobservable buses and the buses connected to it via transformers. $\mathbf{M}_{\mathbf{d i a g}}$ is a diagonal matrix given as:

$$
\mathbf{M}_{\text {diag }}=\left[\begin{array}{llllll}
1 & 0 & 0 & 0 & 0 & 0 \\
0 & 2 & 0 & 0 & 0 & 0 \\
0 & 0 & 3 & 0 & 0 & 0 \\
0 & 0 & 0 & . & 0 & 0 \\
0 & 0 & 0 & 0 & . & 0 \\
0 & 0 & 0 & 0 & 0 & \mathrm{~m}
\end{array}\right]
$$

$I_{5}$ Matrix selects the island which can make all the transformer connected buses to the unobservable bus observable simultaneously. Otherwise the unobservable bus is not moved. Island matrix is updated $\mathrm{I}^{r}$ as follows:

$$
\mathbf{I}^{s}=\mathbf{I}+\mathbf{I} \times \mathbf{P I}
$$


where

$$
\mathrm{PI}=\left\{\begin{array}{c}
1_{j} \tilde{\mathrm{I}}=\text { unobservable bus and transformer connected buses } \\
\hat{\mathrm{j}}=\mathrm{PMU} \text { bus as shown by } \mathrm{I}_{\mathrm{S}} \\
-1 ; \mathrm{i}=\mathrm{j}=\text { unobservable bus plus transformer buses } \\
0 ; \text { Otherwise }
\end{array}\right.
$$

Constraints are then checked for the islands as:

$$
\mathrm{C}_{\mathrm{n}}=\mathrm{I}^{f} \times[\mathrm{PG}-\mathrm{PL}]
$$

PG and PL are $\mathrm{n} \times 1$ vectors of minimum generations and loads of the buses. $\mathbf{C}_{\mathrm{n}}$ is a $\mathrm{m} \times 1$ matrix. Positive elements in $\mathbf{C}_{n}$ indicate the constraint violation for respective Island. NB matrix is formed as shown:

$$
\mathrm{NB}=\mathrm{C} \times\left[\mathrm{I}_{\bmod }-\mathrm{I}_{\bmod }\right]
$$

Here $\mathbf{C}$ is having all its elements equal to one and $\mathbf{I}_{\bmod }{ }^{b}$ and $\mathbf{I}_{\bmod }$ are modified Island matrices after merging all transformer connected buses. If constraint of (18) is violated for an Island, minimum number of buses which must be retained in the original Island to full fill the constraints can be found by solving following problem by binary integer programming:

\section{$\min \quad \mathrm{NB} \times \mathbf{R}$}

$$
\text { subject to } P_{0} \times R \geq E
$$

where

$\mathbf{R}$ is solution vector

$$
\begin{aligned}
& \mathbf{P}_{\mathbf{O}}=\left[\mathbf{I}_{\mathbf{m o d}}{ }^{\prime}-\mathbf{I}_{\mathbf{m o d}}\right] \times \operatorname{diagonal}\left(\left[\mathbf{P G}_{\mathbf{m o d}}-\mathbf{P L}_{\mathbf{m o d}}\right]\right) \\
& \mathrm{E}_{\mathrm{i}}= \begin{cases}0 & ; \text { if constraints for Island i are not violated } \\
\mathrm{PG}_{\mathrm{i}}-\mathrm{PL}_{\mathrm{i}} & ; \text { if constraints for Island i are violated }\end{cases} \\
& \mathrm{PG}_{\mathrm{i}}=\text { sum of all the minimum generations in ith Island } \\
& \mathrm{PL}_{\mathrm{i}}=\text { sum of all the loads in ith Island }
\end{aligned}
$$

where $\mathbf{P G}_{\mathrm{mod}}$ and $\mathbf{P L}_{\mathrm{mod}}$ are again $\mathrm{m} \times 1$ vectors of the equivalent minimum generated power and loads of the buses after merging the buses connected by transformers.

If an element of solution vector $\mathbf{R}$ is 1 , corresponding bus is moved back to its original Island.

2.3.2 The unobservable bus is a central bus or cannot be moved and PMU installed bus is not a central bus

If unobservable bus is a central bus or it is connected to a central bus via transformer, it cannot be moved to neighbouring island for restoring bus observability. Also, if the constraints described in Case A are violated, the unobservable bus cannot be moved to other islands. In such conditions, the PMU installed non-central buses of the neighbouring islands, which can make such buses observable, are brought in the islands of unobservable buses. To do so, all the steps of Case I are implemented in these movements with one extra constraint check.

Island selectivity matrix $I_{5}$ is formed from (8) with $A_{\mathrm{uz} 2}$ rows are now corresponding to PMU installed buses (which can restore observability of unobservable buses) and the buses connected to PMU installed buses via transformers. $A_{u 2}$ Columns now contain the unobservable buses. Accordingly PI matrix is modified as: 


$$
\mathrm{PI}=\left\{\begin{array}{c}
1 ; \mathrm{i}=\text { PMU bus \& } \mathrm{t} / \mathrm{f} \text { connected buses, } \\
\mathrm{j}=\text { unobservable bus as shown by } \mathrm{I}_{\mathrm{S}} \\
-1 ; \mathrm{i}=\mathrm{j}=\mathrm{PMU} \text { installed bus and transformer buses } \\
0 ; \text { Otherwise }
\end{array}\right.
$$

The same procedure (as described in Case A) is repeated to bring back minimum PMU installed buses to their original islands if the constraints of (18) are violated or if the buses connected to PMU installed buses via transformers are unobservable in new islands.

Finally, it is to be checked that the buses which are directly connected to PMU installed bus (not via transformers), must not become unobservable when PMU bus is moved to another Island to restore observability. i.e. the buses which were earlier covered by the PMU installed bus, must have a backup measurement so that even after the PMU installed bus is sent to another island, these buses remain observable.

To ensure this, the observability matrices $\mathbf{o}$ and $\mathbf{A}_{\mathbf{u}}$ are formed again. Let this new matrix be called $\mathbf{A}_{\text {unew }}$ and the latest previous $\mathbf{A}_{\mathrm{u}}$ matrix as $\mathbf{A}_{\text {uold }}\left(\mathbf{A}_{\mathrm{u} 3}\right)$. All-zero rows are added in $\mathbf{A}_{\mathbf{u n e w}}$ and $\mathbf{A}_{\text {uold }}$ corresponding to all the observable buses.

$$
\mathbf{A}_{\text {udiff }}=\mathbf{A}_{\text {uold }}-\mathbf{A}_{\text {unew }}
$$

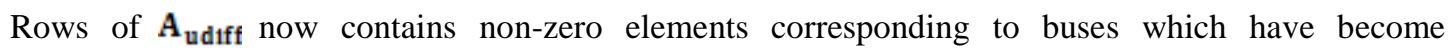
unobservable due to movement of PMU from their parent island to some other Island to restore observability of that Island. All other rows are all-zeros.

To avoid this problem of induced unobservability, such PMUs are called back to their original Island. To minimize the number of PMUs which are to be called back to their original island, the following binary integer programming problem is solved:

$\min \quad$ PMU $\times \mathbf{R}_{2}$

$$
\text { subject to: } A_{\text {udiff }} \times \mathbf{R}_{2} \geq \mathbf{b}_{2}
$$

where $\mathbf{b}_{2}$ is $\mathrm{n} \times 1$ vector having all ones.

Elements corresponding to $1 \mathrm{~s}$ in $\mathbf{R}_{2}$ represent the PMUs which must be called back to their original Islands. Thus, these PMU installed buses are moved to their original Islands and Island matrix I is accordingly modified.

2.3.3 The unobservable bus is a central bus or cannot be moved and PMU installed bus is a central bus:

All the remaining unobservable buses are those which cannot be moved and also, no PMU installed bus can be moved to islands of such buses to restore their observability. In this case, wop is calculated for each island and islands with wop less than 0.9 are found. All such Islands are merged with the Islands which contains PMU buses that can restore observability of unobservable Islands.

This can be done in following simple steps:

- Form observability matrix $\mathbf{o}$ again and calculate weighted observability percentage index wop as given by (9) and (12).

- Form $\mathbf{U}$ matrix again using recent unobservable buses and form a $\mathbf{M}_{\mathbf{U}}$ matrix having islands as the rows and unobservable buses as columns. i.e.

$$
\mathrm{M}_{\mathrm{Uij}}= \begin{cases}1 ; & \text { unobservable bus } \mathrm{j} \text { is in ith island } \\ 0 ; & \text { Otherwise }\end{cases}
$$

- Form Island to Island matrix as follows:

$$
\mathrm{ITI}_{\mathrm{m} \times \mathrm{m}}=\mathbf{M}_{\mathrm{U}} \times \mathbf{U} \times \mathbf{I}^{\mathrm{T}}
$$


The islands $\mathrm{i} \& \mathrm{j}$ are merged if $\operatorname{ITI}_{\mathrm{ij}}=1$.

\section{Case Study}

The method of managing the unobservable buses and the difference between originally reported and the proposed algorithm may be explained by taking an example of islanding of IEEE 57 bus system.

For IEEE 57 bus system when initial islands are formed (Fig. 1) using the original method, unobservable buses, as shown by matrix $\mathbf{U}$ are 2, 9, 14, 17, 21, 31, 37 and 48 . These unobservable buses are covered by PMUs of other Islands at buses 1, 13, 13, 1, 22, 32, 39 and 36 (both in the same island) and 47 respectively. The initial Islands are shown in Fig. 1.

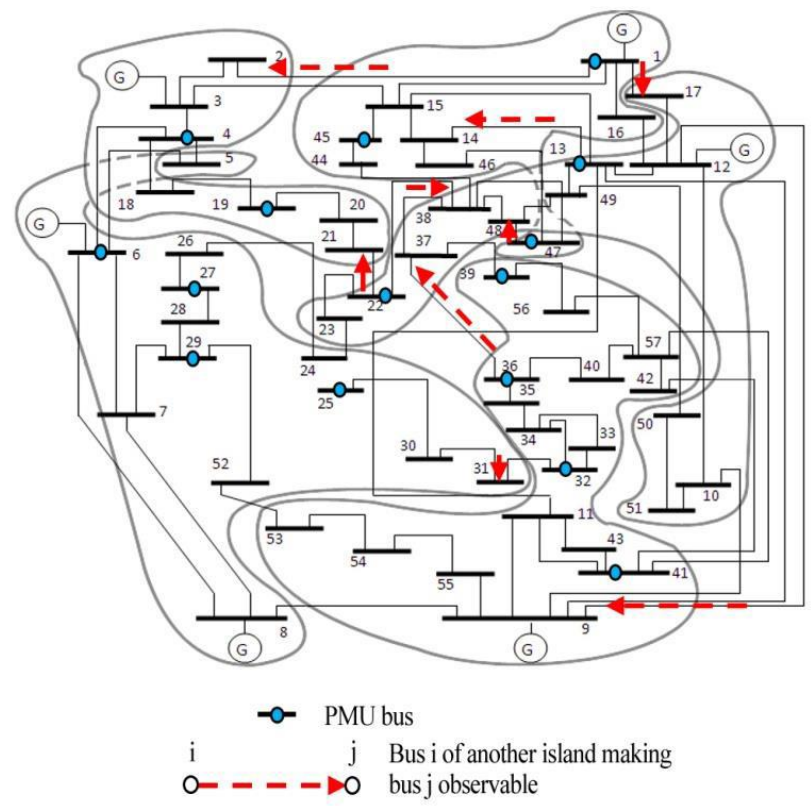

Fig. 1. IEEE 57 bus system initial islands with infinite channel PMUs.

Bus 2 is moved to the island of bus 1, Bus 17 is moved to the island of bus 1, Bus 31 to the island of bus 32 , bus 37 is moved to the island of buses 39 and 36, and bus 48 to the island of bus 47 . Bus 14 is connected to bus 46 via transformers, they must be moved together. Bus 14 is made observable by PMU at bus 13. Using original algorithm, this movement (in the island of bus 13) makes bus 46 unobservable as it has no measurement to cover in new island. Thus inducing un-observability and island wop will decrease. In the proposed improvement, this movement will not be executed. Similarly bus 21 is moved to the island of bus 22 . Since bus 20 which is connected to bus 21 via transformer is unobservable in new island, this will also decrease observability in new island.

Bus 9 is a central bus and cannot be moved to make it observable. PMU installed bus 13 which is a non-central bus is to be brought in the island of bus 9 to cover it. The original algorithm executes this movement without realizing that the bus 13 is covering many other buses in its original island (bus 12,14 and 49) to make them observable. Such a movement will affect the observability of buses which do not have any backup measurements. So bus 12, 14 (newly brought bus) and 49 will be unobservable due to this movement. In the proposed algorithm such movements are denied. Also, movement of buses to any constraint violation of generation-load is not optimized in the original algorithm. Buses 37 and 48 are called back to their islands due to load-generation constraint violations. To make these buses observable PMU buses 36 and 48 are brought to their islands without taking into account that the buses which PMU at 36 and PMU at 48 were covering earlier (46, 35 and 40) will become unobservable due to the movements of bus 36 and 47 in new island thereby inducing un-observability in original islands (Islands 
of buses 36 and 47). This movement will also be successfully checked in the proposed new algorithm.

The final islands for the 57 bus system formed by the proposed algorithm are shown in Fig. 2.

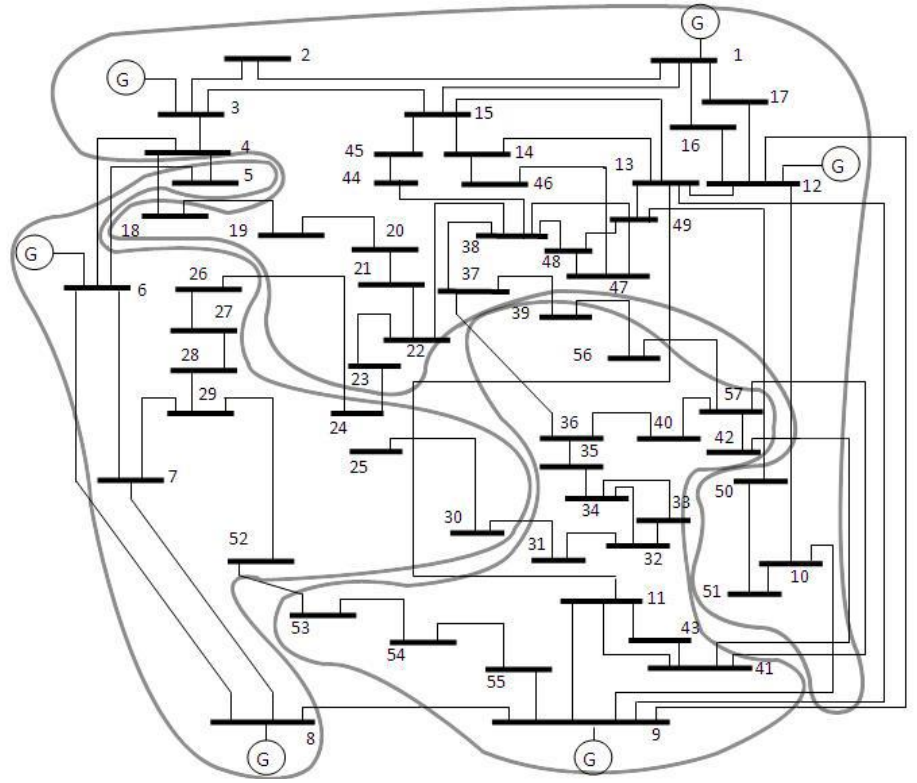

Fig. 2. IEEE 57 bus system final islands with infinite channel PMUs.

Unobservable buses, as obtained by $\mathbf{U}$ matrix are 2, 9, 14, 17, 31, 37 and 48 . These unobservable buses are covered by PMUs of other Islands at buses 1, 13, 13, 1, 32, 39 and 36, and 47 respectively as obtained by Au Matrix. Bus 2 is moved to the island of bus 1, Bus 17 is moved to the island of bus 1 , Bus 31 to the island of bus 32, bus 37 is moved to the island of buses 39 and 36, and bus 48 to the island of bus 47 .

Bus 14 is a non-central bus and is not connected to any central bus via transformers and is hence free to move. Since bus 14 is connected by a transformer to bus 46 and this bus is not observable in the island of PMU installed bus which can make bus 14 observable, the pair (14 and 46) is not moved to new island. Similarly bus 21 is not moved to the island of bus 22 . Since bus 20 which is connected to bus 21 via transformer is unobservable in new island.

Buses 37 and 48 are called back to their islands due to load-generation constraint violations. Optimization is done to call back buses to their respective islands to satisfy load constraints. This optimization will be very helpful in case of distribution system which is having a large number of buses.

Next step is to call PMU installed buses to the islands of unobservable buses $(9,14,21,37,48)$ since these movements are not possible. Bus 13 can make bus 14 observable but is not moved since bus connected to it via transformer (bus 49) in original island is unobservable in new island. Similarly, for the same reason, bus 13 is not moved to the island of bus 9 to make it observable. Also, buses 36 and 47 are called back to their original island after attempts of making buses 37 and 48 observable as their movement will make those buses unobservable (46, 35 and 40) which were earlier covered by them in original islands since they do not have any backup measurement. Similarly bus 22 is called back to its original Island so that observability of bus 23 is not affected.

In order to test the suitability of the proposed algorithm to practical power networks a part of the Indian power grid has been considered. The Indian power grid is geographically divided in five regions and the considered network is a part of the Northern region of the Indian grid.

The network under consideration consists of 75 buses which include $220 \mathrm{~V}$ and $400 \mathrm{~V}$ buses. After application of the proposed algorithm the 75 bus network can be successfully sectionalized into a number of islands ensuring the observability of individual islands. The final islands of 75 bus system after application of islanding algorithm are shown in Fig. 3. 


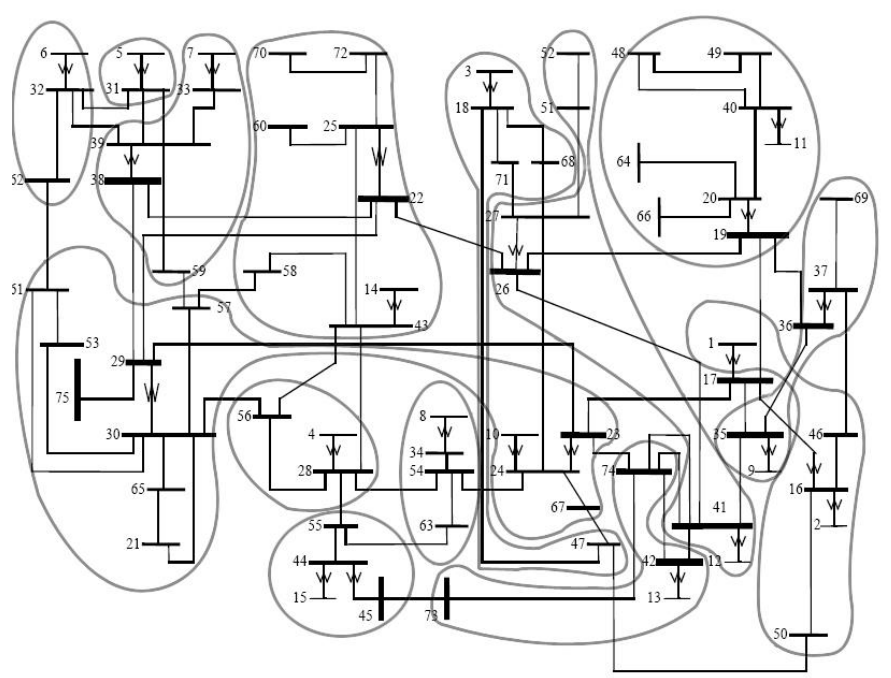

Fig. 3. Indian 75 bus system final islands with infinite channel PMUs.

\section{Effect of PMU Channel Capacity on Islanding}

As discussed in the earlier sections, consideration of PMU channel capacity should be an integral part of any PMU placement scheme. In this work, PMU channel capacity implies the number of buses it can make observable in addition to the bus at which it has been installed. The channel consideration is incorporated in equation (8). Each row in $\mathbf{A}_{\mathrm{I}}$ represents a bus and corresponding non-zero entries are the buses which are connected to this bus. The modified connectivity matrix for finite channel PMU is formed by replacing rows of normal connectivity matrix with the possible combination of channels which a PMU can monitor [7]. Number of combinations is found to be ${ }^{\mathrm{I}} \mathrm{C}_{\mathrm{k}}$ where 1 is the total number of branches incident on that bus and $\mathrm{k}$ is the channel capacity of PMU on that bus. To incorporate channel capacity, $\boldsymbol{A}_{\mathrm{I}}$ matrix formed as:

$$
A_{\mathrm{Ij}}=\left\{\begin{array}{l}
1 ; \text { if a PMU at jth bus is covering ith bus } \\
0 ; \text { otherwise }
\end{array}\right.
$$

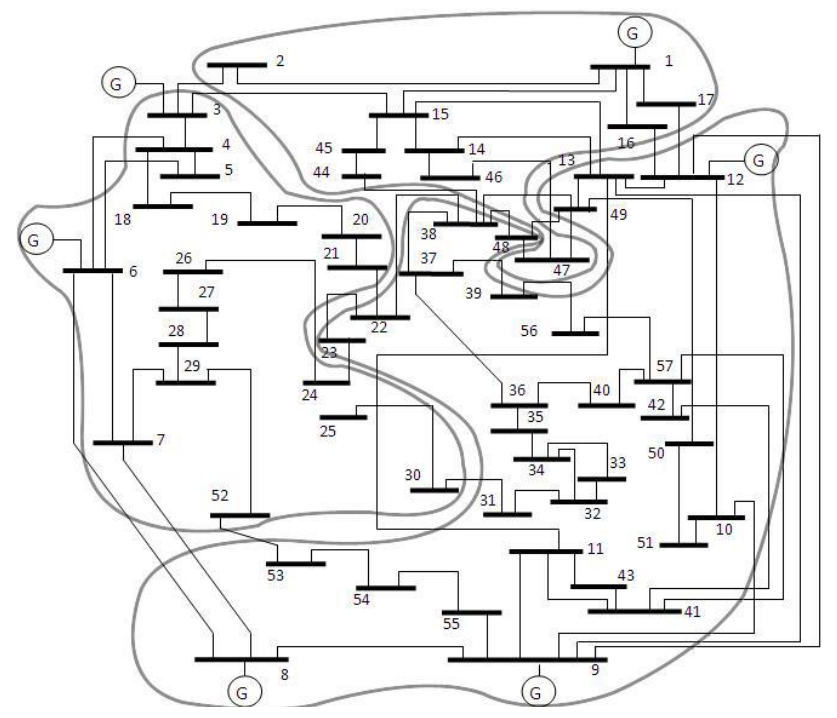

Fig. 4. IEEE 57 bus system final islands with three channel PMUs. 
Whether a PMU is covering a bus or not can be found by running optimal finite channel capacity PMU placement solution as described earlier. The effect of PMU channel capacity on the islanding of IEEE 57 bus system has also been examined. The sectionalized network after the implementation of algorithm for 3 channel PMU is shown in Fig. 4.

It was found that selection of a higher channel PMU do not offer any significant benefits in terms of number of islands and their observability. An extensive study was performed to verify these results further by applying the algorithm to IEEE 118 bus system and the 75 bus system of Indian grid also. The results reveal that the number of islands and their wop becomes constant after a channel limit of 3 or 4 .

Due to space constraints all these results and the sectionalized networks are not presented here. However as a representative case the final islands for 75 bus system for a channel limit of 3 are shown in Fig. 5.

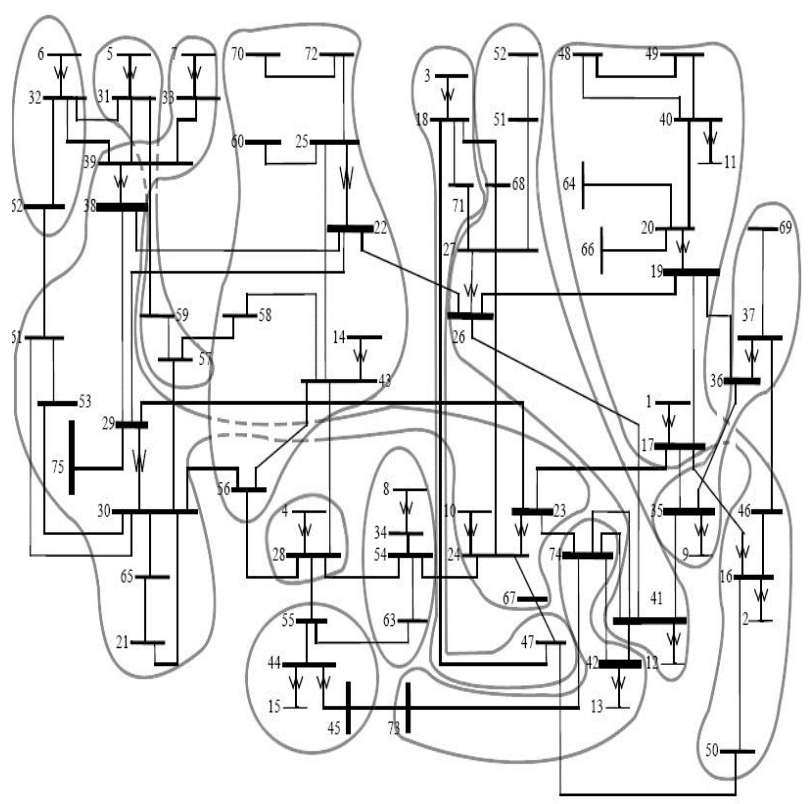

Fig. 5. Indian 75 bus system final islands with three channel PMUs.

These results are in conformity with the earlier reported results regarding optimal PMU placement where it has been shown that higher channel PMUs do not offer any added benefits. Therefore while selecting the number of channels for a practical PMU placement scheme, in general, a channel capacity of 3 or 4 may be sufficient and economic option.

\section{Conclusion}

This paper explored the utilization of phasor measurement units in intentional islanding, A previously reported algorithm for intentional islanding in the presence of PMUs has been considered and improvements have been proposed in the algorithm. These improvements have been highlighted Using case studies on IEEE 57 bus network and a network of the Indian grid. The proposed algorithm has been shown to be an improvement over the original algorithm in terms of applicability and efficiency (in terms of higher value of wop for the resulting islands.

Finally the effect of PMU channel capacity on islanding scheme has also been studied. Based on an extensive study it has been found that the effect of channel capacity on the islanding algorithm does not provide a specific pattern regarding the number of islands or the value of wop for resulting islands. However it has been observed, in general, that higher channel PMUs do not offer any specific benefits in terms of intentional islanding and considering the financial aspects, lower channel PMUs may be applied for implementing effective islanding in the power grid. It is expected that these results will be helpful for 
different PMU manufactures and utilities around the world working in the area of PMU installation in the power grid to make it smart.

\section{References}

[1] Sarmadi SAN, Dobakhshari AS, Azizi S, Ranjbar AM. A sectionalizing method in power system restoration based on WAMS. IEEE Trans. Smart Grid, Mar. 2011; 2(1):190-197.

[2] Nezam-Sarmadi SA, Nouri-Zadeh S, Ranjbar AM, Pishvaie MR. An islanding algorithm to restore a PMU installed power system. In: Proc. the Asia Pacific Power and Energy Engineering Conference, 2010:1-4.

[3] Rihan M, Ahmad M, Beg MS, Anees MA. Robus and economical placement of phasor measurement units in Indian smart grid. In: Proc. the IEEE Innovative Smart Grid Technologies - Asia (ISGT Asia), 2013:1-6.

[4] Gou B. Generalized integer linear programming formulation for optimal PMU placement. IEEE Trans. Power Syst., Aug. 2008; 23(3):1099-1104.

[5] Nourizadeh S, Sarmadi SAN, Karimi MJ, Ranjbar AM. Power system restoration planning based on wide area measurement system. Elsevier Journal Electric Power and Energy Systems, 2012; 43:526-530.

[6] Chaturvedi DK. Soft Computing: Techniques and Its Applications in Electrical Engineering, Springer-Verlag Berlin Heidelberg; 2008; 1.

[7] Kumar R, Rao VS. Optimal placement of PMUs with limited number of channels. In: Proc. the North American Power Symposium, 2011:1-7. 